\title{
A Contrastive Study of English and Chinese Empty Categories
}

\author{
Jing Yang \\ School of Humanities, Tianjin Polytechnic University, Tianjin, China \\ Email:2487682064@qq.com
}

How to cite this paper: Yang, J. (2018) A Contrastive Study of English and Chinese Empty Categories. Open Access Library Journal, 5: e4860.

https://doi.org/10.4236/oalib.1104860

Received: August 21, 2018

Accepted: September 8, 2018

Published: September 11, 2018

Copyright $\odot 2018$ by author and Open Access Library Inc.

This work is licensed under the Creative Commons Attribution International License (CC BY 4.0).

http://creativecommons.org/licenses/by/4.0/

(c) (i) Open Access

\begin{abstract}
This thesis makes a comparative study of English and Chinese empty categories on the basis of Government theory, Binding theory and Control theory. The thesis aims at probing into the overall properties of ECs of the two languages as well as digging out the syntactic and semantic representations in English and Chinese ECs. It also has some reference value for second language acquisition.
\end{abstract}

\section{Subject Areas}

Education, Linguistics

\section{Keywords}

English and Chinese Empty Categories, Government Theory, Binding Theory, Control Theory

\section{Introduction}

In 1981, Chomsky proposed the Government and Binding Theory in his book Lectures on Government and Binding [1], which is the major theory of modern syntactic study. He introduced the significant concepts of principles and parameters [1]. Since it has a strong interactive function, more and more linguists have employed the GB theory to explore the syntactic problems. In the 1990s, this theory developed into the Minimalist Program (short for MP) [2], which is a brand new theory. However, a lot of syntactic problems cannot be solved by the immature theory, so we still employ the GB theory to do the research rather than the latest Minimalist Program.

The study of empty categories has become a hot issue in modern linguistics. Empty categories refer to those elements that don't have the forms of phonetics 
but perform syntactic and semantic functions [3]. Chomsky proposed that the study of these covert elements could help us to better understand the "determining properties of syntactic and semantic representations and the rules that form them" (Chomsky 1981a: 55) [1]. Therefore, the Empty categories will lead to invaluable insights into the nature of human languages.

Empty Categories exist in both English and Chinese [4]. They are complicated and there are similarities and differences in the two languages [5]. So far there are many studies on English and Chinese ECs [4], but little researches adopt the comparative method. Therefore, this thesis attempts to adopt the comparative method to analyze English and Chinese ECs by using typical sentences so as to help readers better understand empty categories.

The study will make a comparative study of English and Chinese Empty Categories within the framework of GB theory, aiming to explain the similarities and differences between English and Chinese ECs. Through the comparative analysis of English and Chinese ECs, we can not only find out the overall properties of ECs of the two covert languages, but also we can dig out the syntactic and semantic representations in English and Chinese ECs.

\section{Literature Review}

The study of Empty Category has been one of the focal topics in linguistics and studied by more and more linguists. This section will focus on the study of Empty Categories at home and abroad.

\subsection{Studies on Empty Categories Abroad}

Chomsky introduced the notion of Empty Categories in 1980s, holding that syntactic structures including the overt and covert elements [1]. Covert elements bear no forms of phonetics but play important role in syntax, these elements are named as Empty Categories [3]. In Chomsky's words, the study of such constituents can help us to better understand the "determining properties of syntactic and semantic representations and the rules that form them" (Chomsky, 1981:55) [1]. Chomsky also classified EC into four types, PRO and pro, NP-trace, Wh-trace. [1] There are two main classification standards: first is its generative mechanism, second is its relationship with the Lexical Categories [2]. At the same time, he put forward the soul of Empty Categories, called Empty Category Principles [1] [2] [3]. Therefore, Empty Category Principle is the core standard that defines the existence of empty categories.

In 1997, Andrew Radford gave the definition of Empty Category as follows: Empty Category refers to an element that lacks of phonological input and establishes a relationship to an antecedent [5]. They have no overt phonetic form, and hence are inaudible or silent (Radford, 1997:131) [5]. He agreed with Chomsky's theories and proposed that Empty Categories are very crucial in the grammar of English [6]. He put forward that all nominals are D-projections, which are comprised of either an overt or covert pronominal determiner (like she or PRO) used without a complement, or an overt or covert pronominal determiner (like 
the or $\Phi$ ) used with a noun expression as its complement [6]. He held that there are significant parallels between the clauses and nominal argument [5].

\subsection{Studies on Empty Categories in China}

Xu Liejiong had done excellent job in the study of EC. In his representative book Free Empty Categories [7], he argued that the four types of ECs defined by Chomsky is not applicable for Chinese. He maintained that Chomsky's typology is not reasonable and those ECs in Chinese should be identified as another kind of EC, that is Free Empty Categories (FEC) [7], which is "characterized by their complete freedom of preferentiality". He defined the typology of EC as follow:

Empty Category [7]

Type 1: EC without specified features: FEC

Type 2: EC with specified features [1] [2] [7]

a) [+anaphor, -pronominal]: NP-trace

b) [-anaphor, +pronominal]: pro

c) [+anaphor, +pronominal]: PRO

d) [-anaphor, -pronominal]: variable (Wh-trace).

$\mathrm{Xu}$ (1986) [7] said there is no distinction between Chinese finite and non-finite clauses and the reference of PRO is decided by the lexical meaning of matrix verbs.

Huang Zhengde was influenced by Chomsky and explained Chinese in the scope of EC and made great achievements. In 1987, he wrote a thesis Remarks on Empty Categories in Chinese [8], he shown his disagreement with Xu's FEC theory. He held (1987) that there are four types of ECs in Chinese, called NP-trace, Wh-trace, PRO and pro [8]. But he classifies the later two into one type in order to better understand the characteristics of Chinese. He also proposed the Generalized Control Rule to identify the distribution and reference of the type of ECs [8] [10].

Shen Yang's study is very valuable on the complete explanation of Chinese ECs. He (1994) proposed three basic principles named Pre-NP Principle, NP-Principle and Post-NP Principle to explain the abstract structures of verbs [9]. The first principle means that there must be an NP before a verb; the second principle indicates that any NP can enter SP without any constraint on its semantic meaning; the third principle means that all the NPs except for Pre-NP can appear the position after verbs [9]. He also put forward Chinese ECs can be classified into three types in modern linguistics, namely ellipsis, trace and proform [9] [10].

Chomsky's study on ECs explored a new field of linguistics. On the basis of his study, more and more linguists and scholars from different countries tried to apply the findings into their native languages and made outstanding contributions on ECs.

\section{Theoretical Basis}

This chapter disposes of theoretical basis of this thesis, contains two parts. Part 
one is about GB theory, part two will focus on control theory and PRO.

\subsection{Government Theory}

The Government Theory is frequently used to study the various modules of grammar, it is a kind of unifying role (Chomsky, 1995:30) [10]. Chomsky proposed that $\alpha$ governs $\beta$ if $\alpha$ c-commands $\beta$ and there is no category $\gamma$ that "protects" $\beta$ from government by $\alpha, \gamma$ protects $\beta$ in this sense if it is c-commanded by $\alpha$ and either (a) or (b) are held (Chomsky, 1995: 79) [10].

(a) $\gamma$ is a barrier dominating $\beta$.

(b) $\gamma$ intervenes between $\alpha$ and $\beta$.

Government has two main categories, antecedent government of $\alpha$, and head government of $\alpha$ by a head, these categories called proper government [1] [10]. According to Minimalist Program, minimize and economy are the principle [2], therefore Chomsky eliminated the intervention condition and restricted the definition of Government theory as $\alpha$ governs $\beta$ if $\alpha$ c-commands $\beta$ and there is no barrier for $\beta c$-commanded by a [10]. This theory is useful to explain the distribution of Empty Category. Huang held that EC can be classified from the point of view whether it is in the governed position (Huang, 1984: 540) [11].

EC be governed-trace $t$

not be governed-PRO

\subsection{Binding Theory}

The Binding Theory is a sub-theory of GB Theory (Chomsky, 1981) [1]. Its start point is tried to explain certain semantic relations among sentence elements. The basic idea is that from the syntactic structure relationship of sentence components to seek the constraints of structure relations on semantic relations [1] [2] [3]. This theory refers to the control and restraints of a certain component of a sentence to the meaning of another component [1] [2]. Specifically, the Binding theory mainly discusses the indexing relationship between the two noun phrases in a sentence.

The Binding Theory is represented by the following principles: [1] [2] [12]

1) An anaphor must be bound in a local domain

2) A pronominal must be free in a local domain

3) An R-expression must be free

In the three principles, anaphor includes reciprocals and reflexives [1] [2], their referential relationship is dependent on the components of the sentence and form the indexing relationship. Pronominal doesn't have independent referential meaning, their fixed referential components cannot be determined by the elements outside the sentence. R-expression called referring expression [1] [2], speakers use such words to refer to people or things in the objective world or context, listeners also know what we are referring to. They have independent referential meaning.

The Binding theory plays a similar role in covert NPs. Firstly, NP-trace is equivalent to anaphor, they have similar properties and must be bound in a local 
domain (Huang, 1984: 550) [11]. Secondly, Wh-trace is equivalent to r-expression [11], it must be free. Thirdly, pro is equivalent to pronominal [11], it must be free in a local domain. Although pro doesn't exist in English, in many other language there is pro.

\subsection{Control Theory}

For PRO, it is neither an r-expression nor a pronoun or anaphor, it seems to be a collection of various characteristics. It shouldn't be explained by binding theory, syntactic linguists chose the control theory to explain PRO. Control Theory is concerned with two issues: the distribution and interpretation of PRO [12] [13]. This theory can be divided into two types according to the meaning of PRO, first type is called arbitrary control; second type has two ways to describe: obligatory control and non-obligatory control [14]. In 1980, Williams put forward the distinction between obligatory control structures and non-obligatory control structures [15]. PRO in obligatory control required an antecedent, while non-obligatory control structures involve a PRO needs not have binders (Franks, S \& N, Hornstein. 1992: 41) [16]. According to the location of control, there are two types of control: subject control and object control. Subject control means that the controller is the subject. Object control indicates that the controller is the object [14]. Take the example:

1: $\mathrm{John}_{\mathrm{i}}$ is reluctant $\mathrm{PRO}_{\mathrm{i}}$ to be well-behaved.

2: Jane ${ }_{j}$ ordered $\mathrm{John}_{\mathrm{i}} \mathrm{PRO}_{\mathrm{i}}$ to be well-behaved.

From the above two sentences, PRO is controlled by John. The first sentence is subject control, the second is object control. In the second sentence, PRO is not coindexed with Jane. Both Jane and John may be controllers, but from the perspective of structure, John is closer to the PRO, so PRO is controlled by John. This distance relationship is expressed most accurately by Rosenbaum's Minimal Distance Principle (1967), which is shortened to MDP.

Minimal Distance Principle (MDP)

PRO is controlled by the closest c-commanding referential NP [14].

There are some examples to refute the Principle, but the thesis will not list and explain it. That is to say, at this stage, no completely satisfactory control theory to explain all the cases.

\section{A Contrastive Analysis of English and Chinese ECs}

The chapter deals with the comparative analysis of English and Chinese ECs and focuses on the classification and generation of the two languages. At the same time, this chapter attempts to explore the similarities and differences between English and Chinese ECs.

\subsection{Properties of English EC}

According to Chomsky's classification of EC, there are three types of EC in English: NP-trace, Wh-trace and PRO, there is no pro in English [1]. And there are 
four types of EC in Chinese: NP-trace, Wh-trace, PRO and pro [8].

\subsubsection{NP-Trace}

NP-trace is formed as the consequence of movement, it appears in passive sentences and raising structures [12] [13] [14].

\section{1) NP-trace in Passive Sentences}

(1a) Police arrest John.

(1b) John was arrested.

D-structure of the sentence: (2a) was arrested John

S-structure of the sentence: (2b) John was arrested $t$

As shown in above two sentences, passive sentence (1b) is the result of conversion from the active sentence (1a), and an empty position is left after the patient John is moving to the beginning of the sentence. From the point of view of $\mathrm{D}$-structure and S-structure, we can see the sentence (2b) is the S-structure of the sentence (1b) and it is the $a$-movements from the D-structure in sentence (2a). The $t$ position, or the position of the complement of arrest in (2b) is where such empty category exist. Therefore, during the generation of the passive sentence, John moved from the complement of D-structure to the subject of S-structure, leaving the trace and forming the type of empty category. This process is called movement analysis, the moving part is called target of movement, the destination is called landing site [17]. In the sentence (2a), John is the target of movement, and the position of its subject is landing site. The reason for the generation can be explained by the case theory. That is verbs in passive voice only allocate case to the position of subject, nor allocate case to the position of complement. Therefore, in order to obtain the case, John moves from the complement position to the subject position.

\section{2) NP-trace in Raising Structures}

NP-trace not only exists in passive sentences, but also in raising structures. There are some verbs and adjectives such as seem, appear, likely, etc, namely raising verbs and raising adjectives [18]. They have the function to move the noun phrases in complement clauses to the position of subject, the process is called NP raising.

(3a) Alex ${ }_{i}$ seems $t_{i}$ to know nothing about it.

(3b) seems Alex to know nothing about it.

From sentence (3b), the raising verb seems move the noun phrase Alex to the beginning of the sentence, and left NP-trace before the infinitive to know nothing. Similar to NP-trace in passive sentences, the NP-trace in raising structures also moved from the lower clause to the subject position, they both influenced by the case. The inflection and raising verbs don't have the ability to allocate case to the noun phrase. In order to get the case, the noun phrase Alex have to move to the subject position of the sentence, leaving the empty position called NP-trace.

(4a) Mary is likely $t_{i}$ to be happy.

(4b) is likely Mary to be happy. 
From the above sentences, the raising adjective likely enables the noun phrase Mary to move to the beginning of the sentence, and left an empty position before the infinitive to be happy. Like raising verbs, raising adjective is disable to allocate case. Therefore, in the sentences, the noun phrase Mary cannot get the case in the infinitive to be happy nor the adjective likely. The noun phrase must raise to the position of subject of the sentence so as to obtain the case, and left an NP-trace.

\subsubsection{Wh-Trace}

Like the NP-trace, Wh-trace or variable is also formed by movement [14]. And the differences between NP-trace and Wh-trace is that object and position of movement. The object to be moved are interrogative pronouns marked with wh, such as what, who. The origin of movement is the position of theta, and the destination is the position of complementizer. And also they are distinguished by the feature case, variable is case-marked and NP-trace is not case marked. In English, it is mainly manifested in the generation of wh-question. There are three types of variables: words that are replaced by related words in the related clauses, wh-movement and the trace in topic structures [17].

S-structure: (5a) The girl whom $\mathrm{i}_{\mathrm{i}}$ you were talking with $\left[\mathrm{NP}_{\mathrm{i}}\right]$ is my sister.

(5b) What $t_{i}$ did you read $\left[\mathrm{NP}_{\mathrm{i}}\right]$ ?

(5c) That book $\mathrm{i}_{\mathrm{i}}$ I will not read [NP $\mathrm{e}_{\mathrm{i}}$ ].

D-structure: (6a) the girl you were talking with whom is my sister.

(6b) did you read what

(6c) I will not read that book

From the above sentences, from D-structure to S-structure, the relative word, wh-word and the noun phrase in topic-prominence sentence leave their traces after moving. In sentences (5b) and (6b), wh-word what move from the complement position of the verb read to the position of complementizer and left the trace. The empty position cannot exchange with lexical category so as to ensure that predicate verbs cannot "illegally redistribute" thematic roles at the syntactic position. In sentences (6a) and (6b), the noun phrase that book moves to the topic and left the trace. In English, the position of topic generally only allows specific components and used infrequently. Variable is case-marked, therefore, variable has got its case before moving.

\subsubsection{PRO}

PRO is not the result of movement, but is base-generated, it is also called non-pro-dropping, corresponding to pro (pro-dropping) [12]. PRO distributes only in the subject position of non-finite clauses [12]. In control theory, control can be divided into two types according to the meaning of PRO, first type called arbitrary PRO; second type has two ways to describe: obligatory control and non-obligatory control [14]. According to the location of control, there are two types of control: subject control and object control [14].

(7a) $\mathrm{John}_{\mathrm{i}}$ decided $\left[\mathrm{PRO}_{\mathrm{i}}\right]$ to go by himself.

(7b) John persuaded Mary $\left[\mathrm{PRO}_{\mathrm{i}}\right]$ to go by herself. 
Sentence (7a) means John decided that he goes by himself, PRO in this sentence is co-indexed with the subject John. Therefore, this sentence belongs to subject control, the subject John controls PRO, and it is the antecedent of the PRO. Sentence (7b) means John persuaded Mary that she to go by herself, Pro in this sentence is the same mark of the object Mary, it is not controlled by the subject John. So it belongs to object control, the object Mary controls PRO, Mary is the controller.

(8a) [PRO] to finish the task is difficult.

(8b) It is difficult [PRO] to learn Mandarin well.

In the above sentences, PRO can refer to anyone and doesn't need to rely on controllers. It is not controlled by any element in the sentence, so it is called arbitrary PRO.

\subsection{Properties of Chinese EC}

In the study of Chinese syntactic, Chinese EC is playing an increasingly important role. The flexibility of Chinese syntactic structure is inseparable from the large number of empty categories in sentences. Therefore, in order to reveal the characteristics of Chinese grammatical structure, it is necessary to understand the nature of empty categories. Chomsky's typology on English EC is also applicable to Chinese EC, there are four types of EC in Chinese: NP-trace, Wh-trace, PRO and pro [8].

\subsubsection{NP-Trace}

Similar to English NP-trace, Chinese NP-trace is also generated by passivization and raising processes [17].

1) NP-trace in passive sentences

(9a) Li Laoshi piping le Xiaowang.

Li Laoshi criticized particle Xiaowang.

Lilaoshi criticized Xiaowang.

(9b) Xiaowang bei piping le.

Xiaowang passive criticized particle.

Xiaowang was criticized.

(10a) Bei piping le Xiaowang.

Passive piping particle Xiaowang.

Was criticized Xiaowang.

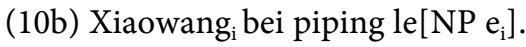

As shown in the above sentences, the passive sentence (9b) is generated from the active sentence (9a). The patient Xiaowang moves to the beginning of the sentence and left the empty position, called NP-trace. Sentence (10a) is the $\mathrm{D}$-structure of sentence $(9 \mathrm{~b})$ and it is the a-movements from the D-structure in sentence (10b). This process is the same with English NP-trace. According to Jaeggli (1986) and Robert (1987), the passive inflection of verbs cannot assign theta-role to the subject position [19]. In (10a) the subject position cannot get the theta-role, the word bei with the property of agent and allocate theta-role to 
the position of subject. In the passive sentence of Chinese, the verb cannot allocate case to the complement position. So the noun phrase Xiaowang moves forward from the argument position to obtain the case, that is to say, the noun phrase is forced by case to appear at the subject position.

\section{2) NP-trace in Raising Constructions}

In raising constructions, the structures and generations of NP-trace in English and Chinese are similar [20]. In Chinese, there are also raising verbs and raising adjectives such as "keneng" (be likely), "kanqilai" (look like) and "kending" (certain), etc. [20]. They are the marks of raising construction in which NP-trace exists. They can cause the noun phrases in the complement clauses to move ahead.

(11a) Ailisi $i_{\mathrm{i}}$ kanqilai $\mathrm{t}_{\mathrm{i}}$ zhidao zhejianshi.

Alice seems know this thing.

Alice seems to know this thing.

(11b) Kanqilai Ailisi zhidao zhejianshi.

Seems Alice knows this thing.

Seems Alice to know this thing.

From sentence (11b), the raising verb kanqilai has the function to make the noun phrase Alice move to the beginning of the sentence, and leaving an empty position before the infinitive zhidao zhejianshi. The NP movement in raising structures are also forced by the case. The inflection is unable to allocate case to the noun phrase, and the raising verb also does not have the ability. The noun phrase in the infinitive or at its original position cannot get the case. Therefore, the noun phrase raise to the subject position so as to get the case, leaving the NP-trace.

(12a) Mali $i_{\mathrm{i}}$ keneng $\mathrm{t}_{\mathrm{i}}$ hui gaoxing.

Mary likely particle happy.

Mary is likely to be happy.

(12b) Keneng Mali hui gaoxing.

Likely Mary particle happy.

Is likely Mary to be happy.

From sentence (12b), the raising adjective keneng enables the noun phrase Mali move ahead, the NP-trace is left behind in the beginning of infinitive huigaoxing after moving. The inflection and adjective never have the ability to allocate case to noun phrase, so the noun phrase Mali cannot obtain case in infinitive nor out of the complement clause. If the noun phrase wants to obtain case, it has to move to the position of subject, leaving the empty position.

\subsubsection{Wh-Trace}

In Chinese, wh-word does not necessarily undergo wh-movement, that is to say, the related movement is not obligatory, but there are traces left after the topicalization movement [18]. Therefore Chinese is a kind of topic-prominence language.

(13a) Xiaoshuo ${ }_{i}$ wo du le $t_{i}$. 
Novels, I read particle.

Novels, I have read.

(13b) Naben xiaoshuo $\mathrm{o}_{\mathrm{i}}$ wo du le $\mathrm{t}_{\mathrm{i}}$.

That novel, I read particle.

That novel, I have read.

In (13a), the noun Xiaoshuo moves to the topic and leaves the trace, referring to all the novels I have read. In (13b) the noun Naben xiaoshuo moves to the topic and leaves the trace, referring to that novel I have read. This kind of trace generated from the movement of topic, and marked with $t$, which is co-referenced with the moving part, so we mark $\mathrm{t}_{\mathrm{i}}$.

(14a) Wo de bao zai nali?

My bag is where?

Where is my bag?

(14b) Nali wo de bao zai?

Where my bag is?

In (14b), the sentence is ungrammatical, because the wh-phrase Nali cannot move to the position of complimentizer. That is to say, in Chinese the wh-words or wh-phrases do not necessarily undergo wh-movement and can locate in-situ, they can keep in the position of base-generated.

(15a) Mama xiangxin wo maile shenme.

Mother believes I bought particle what.

Mother believes that what I bought.

(15b) Mama xiangzhidao wo maile shenme.

Mother wants to know I bought particle what.

Mother wants to know what I bought.

(15c) Mama wenwo maile shenme.

Mother asked me bought particle what.

Mother asked me what bought.

According to Huang (1984), verbs such as renwei xiangxin carry feature of [-Wh] [11], so they cannot occur at the interrogative clauses. In sentence (15a), the wh-word shenme appears after the verb renwei, because the verb renwei carry feature of $[-\mathrm{Wh}]$, so this sentence is ungrammatical. While verbs like wen xiangzhidao carry feature of $[+\mathrm{Wh}]$, they must occur at the interrogative clauses. In addition, verbs such as xiangzhidao carry feature of $[ \pm \mathrm{Wh}]$ and they can exist either in interrogative clauses or non-interrogative clauses. Therefore the sentence (15b) and (15c) are grammatical.

\subsubsection{PRO}

PRO is the most mysterious form of empty categories. Due to the low degree of Chinese grammaticalization, the finite and infinite verbs have no obvious marks, the demarcation between finite clauses and nonfinite clauses is not clear [12]. It is difficult to define PRO.

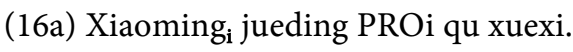

Xiaoming decided to go to study. 


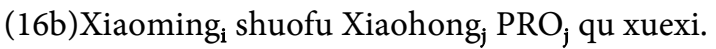

Xiaoming persuaded Xiaohong to go to study.

Sentence (16a) implies that Xiaoming wants himself to study. It is obvious that Xiaoming is the antecedent and controls PRO. So xiaoming is the controller. This is called subject control. Sentence (16b) wants to express that Xiaoming persuades Xiaohong to study. The PRO is controlled by object and it refers to Xiaohong, so Xiaohong is the controller. This is an object control structure. As the two sentences shown, control theory does work in Chinese, but it cannot fully explain Chinese PRO.

(17a) Ta xihuan PRO zai fangjian shuijiao.

He likes to sleep at room.

(17b) Ta jinzhi PRO zai fangjian xiyan.

He forbids to smoke at room.

In the above sentences, it is difficult to decide whether the sentences are subject control or object control. In (17a), PRO may refer to himself or any other people. In (17b), PRO cannot refer to the person himself but any other person. So the empty position may refer freely according to the context.

(18a) PRO xiyan dui jiankang shi buli de.

Smoking is harmful to health.

(18b) PRO Xuexi hanyu henzhongyao.

Studying Chinese is very important.

In the two sentences, PRO can refer to anyone and has no antecedent, it is not controlled by any element in the sentences. This is called arbitrary PRO.

\subsubsection{Pro}

According to Chomsky, pro is used in the subject position of finite clauses that receive case [1]. The use of pro can be regarded as the omission of overt pronouns, called pro-drop. The Chinese language belongs to the pro-dropping language [12], while in English there is no such phenomenon and it doesn't allow a null subject in a finite clause.

(19a) pro Jiang guo hua le.

pro has spoken particle.

pro has spoken.

(19b)Ta shuo pro mingtian huilai.

He said pro tomorrow particle arrive.

He said that tomorrow will arrive.

(19c) pro Qing wu xiyan.

pro Please do not smoke.

Do not smoke, please.

In sentence (19a), the subject exists in the D-structure. The pro refers to an entity in the objective world, and it can refer to anybody. In sentence (19b), pro can be referred to the subject he, or it has its own independent reference. In the two sentences, the subject was omitted because the speaker or hearer knows the reference of the conversation. In different context, it may be referred different 
person, so pro may be determined by the context. In sentence (19c), pro is in the case possessing position and it refers to anyone. The null subject is dropped as its reference is generic and can be understood by people subconsciously.

To sum up, there are similarities and differences of classification and generation between English and Chinese ECs. The similarities mainly lie in NP-trace and PRO, while the differences lie in Wh-trace and pro.

\section{Conclusions}

The purpose of this thesis is to draw an overall picture of the similarities and differences between English and Chinese ECs. By making the comparative analysis of ECs in English and Chinese, this thesis concludes the properties of English and Chinese ECs, and finds out the similarities and differences between them.

The similarities of English and Chinese ECs mainly lie in NP trace and PRO, while the differences lie in pro and Wh-trace. English and Chinese NP-trace are all generated in passive sentences and raising structures. The aim of movement is that NP-trace has to get case at the position of subject. PRO in English and Chinese both occurs in the subject position of non-finite clause, and explained by control theory. Apart from the similarities, there are also differences: Firstly, in English there is no pro while in Chinese it has. Secondly, Wh-trace in English is generated through wh-raising in interrogative clauses. Chinese wh-words or wh-phrases do not necessarily undergo wh-movement and they usually remain in situ. Most of the trace is generated from the movement of the topic of the sentences.

The study is expected to inspire further studies on Empty Categories in English and Chinese.

\section{Acknowledgements}

I'm deeply grateful to my supervisor, Professor Ma Daoshan, whose inspiration and enlightening guidance help me to complete my thesis.

\section{Conflicts of Interest}

The author declares no conflicts of interest regarding the publication of this paper.

\section{References}

[1] Chomsky, N. (1981) Lectures on Government and Binding. Foris, Dordrecht.

[2] Chomsky, N. (1982) Some Concepts and Consequences of Theory of Government and Binding. MIT Press, Cambridge.

[3] Chomsky, N. (1957) Syntactic Structures. Mouton, The Hague.

[4] Ma, D.S. (2016) An Outline of English and Chinese Syntactic Studies. World Book Publishing Co. Ltd., Guangzhou.

[5] Radford, A. (1997) A Syntactic Theory and the Structure of English-A Minimalist 
Approach. Cambridge University Press, Cambridge. https://doi.org/10.1017/CBO9781139166706

[6] Radford, A. (2000) Syntax: A Minimalist Introduction. Foreign Language Teaching and Research Press, Beijing.

[7] Xu, L.J. (1986) Free Empty Category. Linguistic Inquiry, 17, 75-93.

[8] Huang, C.-T.J. (1987) Remarks on Empty Categories in Chinese. Linguistic Inquiry, 18, 321-327.

[9] Shen, Y. (1994) A Study of Modern Chinese Empty Categories. Shandong Education Press, Shandong.

[10] Chomsky, N. (1995) The Minimalist Program. MIT Press, Cambridge.

[11] Huang, C.-T.J. (1984) On the Distribution and Reference of Empty Pronouns. Linguistic Inquiry, 15, 531-560.

[12] Wen, B.L. (2002) An Introduction to Syntax. Foreign Language Education and Research Press, Beijing.

[13] Song, G.M. (1997) Introduction to Syntax. China Social Science Press, Beijing.

[14] Mei, D.M. (2007) Modern Syntax. Shanghai Foreign Language Education Press, Shanghai.

[15] Williams, E. (1980) Predication. Linguistic Inquiry, 11, 203-238.

[16] Franks, S. and Hornstein, N. (1992) Secondary Predication in Russian and Proper Government of PRO. Control and Grammar, 6, 40-45.

https://doi.org/10.1007/978-94-015-7959-9_1

[17] Cao, Q. (2009) A Syntactic and Cognitive Study of Chinese and English Empty Categories. M.A. Thesis, Hunan Normal University, Changsha.

[18] Jiang, T. (2010) A Contrastive Study of English and Chinese Empty Categories from the Perspective of TG Theory. M.A. Thesis, University of Jinan, Jinan.

[19] Jaeggli, O. (1986) The Null Subject Parameter and Parametric Theory. The Null Subject Parameter, 6, 27-39.

[20] Zheng, Y.Y. (2003) A Comparative Study of Empty Categories Between English and Chinese. M.A. Thesis, Shanghai International Studies University, Shanghai. 